\title{
PROOF OF A POLYNOMIAL CONJECTURE
}

\author{
G. K. KRISTIANSEN
}

ABSTRACT. Let a real polynomial have only real roots, all belonging to an interval $I$. An inequality is proved, relating the average value of the polynomial between two consecutive roots to its maximal absolute value in $I$.

In [1] P. Erdös made a conjecture running approximately like this (slightly generalized):

Let $f(x)$ be a polynomial of degree $n(\geqq 2)$ all the roots of which are in the interval $[-1,1]$. The functional

$$
F(f)=\int_{a}^{b} d x|f(x)| /(b-a) \max _{-1 \leqq x \leqq 1}|f(x)|,
$$

where $a$ and $b$ are consecutive roots of $f(x)$, will then assume its maximal value if $f(x)$ is proportional to the Chebyshev polynomial $T_{n}(c x+d)$, where $c$ and $d$ are suitably chosen constants, and $\{a, b\}$ is an arbitrary pair of roots.

Proof. Denote by $P$ the set of polynomials (with real coefficients) of degree $n \geqq 2$, all the roots of which are in the interval [ $-1,1]$. Obviously the subset of $P$ consisting of polynomials of fixed norm is compact. Therefore $P$ contains an optimal polynomial $f$, i.e. $F(f) \geqq F(g)$ for all polynomials $g \in P$. Putting $f_{1}(x)=f(c x+d)$ we get

$$
F\left(f_{1}\right)=\int_{a}^{b} d x|f(x)| /(b-a) \max _{-c+d \leqq x \leqq c+d}|f(x)| .
$$

Denote the roots of $f$, ordered according to the magnitude of their indices, by $\left\{x_{j}\right\}(1 \leqq j \leqq n)$. We have $-1 \leqq x_{1} \leqq \cdots \leqq x_{n} \leqq 1$. If, now, $|f(x)|$ did not assume its maximal value between $x_{1}$ and $x_{n}$, we could set $c=\left(x_{n}-x_{1}\right) / 2$ and $d=\left(x_{n}+x_{1}\right) / 2$, which would give $F\left(f_{1}\right)>F(f)$, a contradiction. But then $f_{1}$ must be optimal if $f$ is, and we can assume $-1=x_{1} \leqq \ldots \leqq x_{n}=1$.

Received by the editors October 2, 1972.

AMS (MOS) subject classifications (1970). Primary 26A75.

(c) American Mathematical Society 1974 
For $n=2$ we have $F(f)=\frac{2}{3}$. Assume $n>2$; then $f$ may have multiple roots. Let us investigate the possibility $a=b$. Let $\left\{f_{m} ; 1 \leqq m<\infty\right\}$ be a fundamental sequence of polynomials in $P$ tending towards $f$. For all $f_{m}$ we require the smallest root to be -1 and the largest to be 1 . Let the roots $b_{m} \rightarrow b$ and $a_{m} \rightarrow a$. If, now, $b=a$, i.e. $b_{m}-a_{m} \rightarrow 0$, we must have

$$
\max _{a_{m} \leqq x \leqq b_{m}}\left|f_{m}(x)\right| / \max _{-1 \leqq x \leqq 1}\left|f_{m}(x)\right| \rightarrow 0
$$

according to the following argument:

We can put $f_{m}(x)=\prod_{p=1}^{n}\left(x-x_{p, m}\right)$, where $x_{p, m} \rightarrow x_{p}$ for $m \rightarrow \infty$. For $a_{m} \leqq x \leqq b_{m}$ we have

$$
\left|f_{m}(x)\right| \leqq\left(\left(b_{m}-a_{m}\right) / 2\right)^{2} \cdot 2^{n-2} ;
$$

for $m$ sufficiently high one of the other root intervals remains greater than $2 / n$. In this interval we have: $\max \left|f_{m}(x)\right|>(1 / n)^{n}$, which is independent of $m$. But, according to [2] we have

$$
\int_{a_{m}}^{b_{m}} d x\left|f_{m}(x)\right|<\left(b_{m}-a_{m}\right) \cdot \max _{a_{m} \leqq x \leqq b_{m}}\left|f_{m}(x)\right| \cdot \frac{2}{3},
$$

so that $F\left(f_{m}\right) \rightarrow 0$ for $m \rightarrow \infty$, a contradiction. Therefore, we can assume $-1 \leqq a<b \leqq 1$.

Since $f$ is optimal we have $F(f+\varepsilon \phi) \leqq F(f)$ for all polynomials $\phi \in P$, for which $f+\varepsilon \phi \in P$ ( $\varepsilon$ is a "sufficiently small" positive number). We can assume that $f(x)>0$ for $a<x<b$, and that $\max _{a<x<b} f(x)=f(z)$, where $a<z<b$.

We let $\phi$ have the same roots as $f$ except for:

(1) \pm 1 , whose multiplicities are decreased by 1 (note that a subsequent linear transformation of the independent variable (preserving the value of the functional) can restore the condition $\left.-1=x_{1} \leqq \cdots \leqq x_{n}=1\right)$;

(2) $a$ (and $b$ ), whose multiplicity is increased by 1 , if $a$ is a simple root, and decreased by 1 , if $a$ is a multiple root;

(3) $z$, which is a double root for $\phi$;

(4) multiple roots $c$ (where $c \neq a$ and $c \neq b$ ), whose multiplicities are decreased by 2 (double roots for $f$ shall not be roots for $\phi$ );

(5) two consecutive simple roots $c$ and $d$ satisfying the condition that $|f(x)|$ does not assume its maximal value for $c<x<d ; c$ and $d$ shall not be roots for $\phi$.

Furthermore, $\phi(x) \geqq 0$ for $a<x<b$.

It is seen that it is possible to choose $\phi \in P$ not identically 0 and satisfying these requirements (making $F(f+\varepsilon \phi)>F(f)$ for $\varepsilon$ sufficiently small) except in the case where $f$ has the form indicated in the theorem. Q.E.D. 


\section{REFERENCES}

1. P. Erdös, Note on some elementary properties of polynomials, Bull. Amer. Math. Soc. 46 (1940), 954-958 (see p. 957). MR 2, 242.

2. P. Erdös and T. Grünwald (Gallai), On polynomials with only real roots, Ann. Math. 40 (1939), 537-548 (see p. 538). MR 1, 1.

Fynsvej 52, DK 4000 Roskilde, Denmark 